\title{
Segregación por género y Formación Profesional: aportaciones al debate sobre la situación actual
}

\author{
Gender Segregation and Vocational Training: \\ Contributions to the Debate on the Current Situation
}

\author{
Juli Antoni Aguado Hernàndez, Francisco J. Cano Montero y $M^{a}$ José Sánchez Pérez ${ }^{1}$
}

\section{Resumen}

En el presente artículo se presenta un estudio sociológico que analiza la situación actual de la segregación de género en la Formación Profesional. Se trata de una etapa educativa con un marcado sesgo de género que se observa en el hecho de que, de las 26 Familias Profesionales existentes, hay 15 claramente masculinizadas, mientras que el alumnado femenino es mayoritario en cinco. Esta circunstancia se traslada a los entornos profesionales a los que conducen estos estudios, generando una segregación horizontal en el mundo laboral.

A partir del análisis de fuentes secundarias de información, se observa cómo, en general, el peso porcentual de las alumnas desciende en todos los niveles de FP, aunque en los últimos cursos su peso se mantiene prácticamente igual. Desde un análisis pormenorizado, con todas las precauciones que hay que tener por las evoluciones divergentes experimentadas por las diferentes Familias Profesionales, en los últimos años se observa un aumento de la matriculación de hombres en los ciclos feminizados sin que se incremente en general el peso de las mujeres en los masculinizados. Esto permite hablar de una escasa o casi nula reducción del sesgo de género en este ámbito.

Posteriormente, se realizan aportaciones al debate sobre las posibles explicaciones del fenómeno analizado y las implicaciones que lleva aparejadas respecto a la elección de los estudios y respecto a las desigualdades entre los géneros en cuanto a la inserción laboral y las condiciones de trabajo de los hombres y de las mujeres. Asimismo, se plantean posibles propuestas para reducir estas situaciones de desigualdad de género.

\section{Palabras clave}

Formación Profesional, segregación horizontal, segregación de género, desigualdad.

\section{Abstract}

This article presents a sociological study that analyzes the current situation of gender segregation in Vocational Training. This is an educational stage with a marked gender bias that is observed in the fact that, of the 26 existing Professional Families, there are 15 clearly masculinized, while the female students is the majority in five. This circumstance affects to the professional environments to which these studies lead, generating a horizontal segregation in the labor world.

From the analysis of secondary sources of information, it is observed how, in general, the percentage of the female students decreases at all levels of VET, although in recent years their rate remains practically the same. From a detailed analysis, with all the precautions that must be taken due to the divergent evolutions experienced by the different Professional Families, in the late years there has been an increase in the enrolment of men in feminized vocational training degrees without the enrolment of women being increased in the masculinized degree. This allows us to speak of little or nule reduction in gender bias in this area.

Subsequently, contributions are made to the debate on the possible explanations of the phenomenon analyzed and the implications that it entails regarding the choice of studies and the inequalities between genders in terms of labour insertion and working conditions of men and women. Women's. Likewise, possible proposals are suggested to reduce these situations of gender inequality.

\section{Keywords}

Vocational training, horizontal segregation, gender segregation, inequality.

\section{Cómo citar/Citation}

Aguado Hernàndez, Juli Antoni; Cano Montero, Francisco Javier y Sánchez Pérez, María José (2020). Segregación por género y Formación Profesional: aportaciones al debate sobre la situación actual. Revista de Sociología de la Educación-RASE, 13 (3), 308-327. http://dx.doi. org/10.7203/RASE.13.3.16583. 


\section{Introducción}

La Formación Profesional (en adelante FP) ha experimentado un crecimiento exponencial en las últimas décadas, tanto en la ingente y variada oferta de ciclos formativos (en adelante CCFF) como en el número de alumnado que la cursa².

Asimismo, la FP ha sido una opción educativa que, tradicionalmente, ha sido considerada masculinizada en tanto que la oferta formativa se centraba, principalmente, en los sectores productivos integrados por hombres. Como consecuencia del crecimiento que ha experimentado el sector servicios, se han producido cambios en la composición de la misma y en relación al alumnado que se matricula en los distintos CCFF.

En la actualidad existe una concentración femenina en torno a cinco familias profesionales orientadas hacia los servicios ${ }^{3}$, con un marcado sesgo de género, y una dispersión masculina por el resto de familias profesionales, aunque concentradas en la industria, la construcción y las nuevas tecnologías.

Asimismo, en general, el peso porcentual de las alumnas desciende en comparación con 2011 en todos los niveles de FP, aunque los últimos cursos su proporción se mantiene prácticamente igual. Desde un análisis pormenorizado, con todas las precauciones que hay que tener por las evoluciones divergentes experimentadas por las diferentes familias profesionales, en los últimos años se observa un aumento de la matriculación de hombres en los ciclos feminizados sin que se incremente en general el peso de las mujeres en los masculinizados, lo que no permita hablar de una tendencia a la reducción del sesgo de género en este ámbito.

Por lo tanto, como afirman Mariño y Rial (2019), la cuestión de los estereotipos y las discriminaciones de género ${ }^{4}$ en la FP es una temática que en la actualidad sigue sin resolverse.

Desde este punto de partida, de forma similar a la idea «campos» en Bourdieu ([1979] 1988, [1980] 1991), las interacciones en el ámbito de la FP pueden entenderse como una red o una configuración de relaciones objetivas de acción e influencia y alianzas de agentes, en el caso de estudio, en la esfera de la formación y entre ésta y el ámbito laboral.

Pero este concepto está ligado al de «habitus», pues la posición de las diferentes entidades y actores en esta esfera dependen, entre otros factores ${ }^{5}$, del conjunto de predisposiciones a la acción por parte de los agentes socializados en las condiciones sociales objetivas; en nuestro caso de estudio, en las prácticas habituales que conducen al mantenimiento de la segregación por género en estos niveles formativos.

En este sentido, sobre la base de los pasos necesarios para llevar a efecto el estudio científico de un «campo» destacados por Bourdieu y Wacquant (2005), la investigación que presentamos, llevada a cabo por profesorado del área de Sociología de la Educación de la Facultad de Ciencias Sociales, en colabora-

2 Con la LOGSE se crean 22 familias profesionales y un total de 142 títulos, con la LOE se establecen hasta 26 familias profesionales y más de 150 títulos. Respecto al alumnado, solo en la última década casi se duplica el número, pasando de 486.893 (curso 2008-09) a 838.764 (curso 2018-19) matriculados.

3 Administración y gestión, Imagen personal, Sanidad, Servicios Socioculturales y a la Comunidad y Textil, Confección y Piel.

4 Según la Oficina del Alto Comisionado de las Naciones Unidas para los Derechos Humanos: «Un estereotipo de género es una opinión o un prejuicio generalizado acerca de atributos o características que hombres y mujeres poseen o deberían poseer o de las funciones sociales que ambos desempeñan o deberían desempeñan. Su utilización consiste en «la práctica de asignar a una persona determinada, hombre o mujer, atributos, características o funciones específicas, únicamente por su pertenencia al grupo social masculino o femenino» (https://www.ohchr.org/sp/issues/women/wrgs/pages/genderstereotypes.aspx). El Comité para la Eliminación de la discriminación contra la Mujer de la ONU, en el artículo 1 de sus estatutos, define la discriminación de género como: «Cualquier distinción o exclusión hecha en base al sexo [y] que tenga el efecto o propósito de disminuir o nulificar el reconocimiento, goce y ejercicio por parte de las mujeres, independientemente de su estado civil, sobre la base de igualdad del hombre y la mujer, de los derechos humanos y libertades fundamentales en las esferas política, económica, social, cultural, civil o en cualquier otra esfera». (https://www.ohchr.org/SP/hrbodies/cedaw/pages/cedawindex.aspx).

5 Como, por ejemplo, el tipo, el volumen y la legitimidad de su capital, sus recursos o su nivel competencial. 
ción con profesorado de la Facultad de Ciencias de la Educación de la Universitat de València, se pretenden diferentes objetivos.

En primer lugar, comprobar si dicha afirmación -la FP como etapa educativa masculinizada- se sigue ajustando a la realidad actual, pues existen indicios que en gran parte esto sigue siendo así.

Por otro lado, describir la distribución por género que se produce en la FP tratando de aportar apuntes para el debate sobre los habitus de los actores de la esfera educativa de la FP, los diferentes sistemas de disposiciones que han adquirido al internalizar un determinado tipo de condiciones de actuación; es decir, explorar algunas explicaciones sobre la segregación por géneros (el proceso de socialización y las sanciones familiares y sociales, la identidad de género, los estereotipos de género, la elección de las trayectorias formativas). Y también de las implicaciones que se puedan derivar de dicha situación para las mujeres (menos probabilidades de trabajar en ocupaciones fuertemente masculinizadas, peores condiciones de trabajo, mayor precariedad laboral y vital y trabajos de menor prestigio social).

Para llevar a buen puerto estos objetivos, el texto comienza con la exposición de la desigualdad de género como estructura social - campo- y en los entornos laborales, para pasar, posteriormente, a la presentación de los resultados sobre la relación entre FP y género y la distribución por género de las diferentes familias que conforman esta formación, continuando con el debate indicado.

Para concluir se exponen posibles propuestas, y algunas de las respuestas que se están dando y se pueden dar, a situaciones no deseadas que puedan traducirse en desigualdad de género, con la finalidad de mejorar las oportunidades laborales de las personas egresadas en los ciclos de FP.

\section{Metodología}

El estudio que se presenta se ha realizado a partir del análisis de fuentes de datos secundarias, así como de investigaciones anteriores de otros autores, relacionados con el tema que nos ocupa.

La información del ámbito estatal se ha extraído de la Estadística de las Enseñanz̧as no universitarias, de la Subdirección General de Estadística y Estudios del Ministerio de Educación, Cultura y Deporte, actualmente Ministerio de Educación y Formación Profesional (MEyFP), y a través de los informes que elabora (Informe Datos y Cifras).

La evolución -el incremento- del alumnado, se han recolectado los datos del MEyFP desde el curso anterior a la crisis (2007-2008) hasta la actualidad; para la distribución por género de las personas egresadas en FP, se ha partido desde el inicio de la LOGSE (curso 1990-1991) y para el análisis pormenorizado por familias profesionales, se han considerado los tres últimos informes, así como el del curso 2011-2012 para tomar perspectiva en el análisis de la información. Para los cálculos se ha considerado el alumnado de los CCFF de enseñanza presencial y a distancia, agrupando los ciclos LOGSE y LOE.

También se ha recurrido a distintos estudios presentados a través de comunicaciones y publicaciones resultado del proyecto de investigación interuniversitario «Mujeres en mundo de hombres» subvencionado por el Plan Nacional de Investigación.

Se trata, por lo tanto, de una investigación descriptiva que persigue una caracterización de la realidad, el estado de la cuestión respecto a la distribución desigual entre varones y mujeres en los estudios pro- 
fesionales, discutiendo sobre relaciones entre factores, pero que tiene una intención futura explicativa buscando vínculos causales entre estos aspectos.

\section{La desigualdad de género como estructura social}

El concepto de «género», puede definirse como «el conjunto de creencias, rasgos personales, actitudes, sentimientos, valores, conductas y datos que diferencian a hombres y mujeres a través de un proceso de construcción social» y se observa en distintas sociedades y períodos históricos, así como en el imaginario colectivo (Martín, 2006: 40). Por tanto, es un producto de la cultura y cada una desarrolla el término de manera diferente.

Asimismo, el género, además de describir un sistema de relaciones sociales jerárquicas -basadas en la diferencia sexual y construidas a través del parentesco-, también funciona como un sistema simbólico que asigna significados a los individuos dentro de una misma sociedad. En este sentido, los roles de género, apoyándose en diferencias sexuales o de tipo biológico, se establecen como normas que determinan lo que es masculino y lo que es femenino. El género se convierte, por tanto, en un criterio de identidad (Amorós, 2000).

Un elemento que también es importante delimitar es el de «estereotipos de género», definido más arriba. Estos, como «fiel reflejo de una cultura y una historia, $[. .$.$] se caracterizan por su fuerte resistencia$ al cambio y por seguir vigentes en la sociedad hasta que se produzca un cambio en los roles de mujeres y hombres». (Mosteiro y Porto, 2017: 153).

En definitiva, lo masculino y lo femenino, el género, son constructos sociales; esto implica, por tanto, que son elementos artificiales creados por el ser humano. Y, por tanto, si es una construcción, puede reconstruirse o, inclusive, deconstruirse.

Pero no puede olvidarse que el término género se construye en relación a otro concepto clave, el de «patriarcado»; es decir, poder o gobierno por parte del padre y, por extensión, de todos los hombres, que pone de manifiesto las relaciones de poder existentes entre géneros (Tubert, 2003). Sistema patriarcal de género que se establece sobre y converge con el sistema económico capitalista (Benería, 2005, 2007), tal como se expone en el próximo apartado. En definitiva, es la configuración de relaciones objetivas de acción e influencia y alianzas de agentes; es decir, el poder que se observa al asignar los roles sociales tanto a las mujeres como a los hombres. Asigna espacios y otorga valor y posee autoridad para nombrar y establecer las diferencias. El género expresa diferencias de poder, pero también las reproduce a través del discurso sobre las diferencias.

El patriarcado y la división sexual del trabajo plantean una característica polifacética y precisa para perpetuarse en el tiempo: «el reconocimiento y la complicidad, en cierto modo, de las mujeres» (Tubert, 2003: 143); las cuales aceptan los modelos de lo femenino como inevitable e incluso necesario. Este conjunto de predisposiciones a la acción por parte de los agentes socializados en las condiciones sociales objetivas: el patriarcado, como señala Cobo (2011), promueve la idea de que la inferioridad es inherente a la naturaleza femenina para mantener un orden natural del sistema. De esta manera se legitima el alejamiento de las mujeres del poder y, a cambio, a través de éste se les asignan unos valores y roles sociales propios, mediante la denominada «mística de la maternidad» (Di Nicola, 1991: 25). 
En este marco, por lo que interesa aquí, las diversas instituciones educativas, a través del currículum oculto, refuerzan los estereotipos de género que se inician en el seno familiar; estereotipos que tienen una gran influencia en el autoconcepto y logro académico (Igbo et al., 2015).

\section{La desigualdad de género en los entornos laborales}

En el mercado profesional este orden patriarcal se traduce en la «división sexual del trabajo», que implica una división jerárquica y binaria del trabajo en base a la cual, los roles socialmente atribuidos y las tareas relacionadas con la producción en el ámbito público son inculcadas a los niños y las asociadas a la reproducción y al espacio doméstico privado son enseñadas a las niñas como algo «natural», propio de su género, y transmitidas mediante mecanismos de poder y control social, sutiles o explícitos (Benería, 2005, 2007; Brunet y Alarcón, 2005). Esta relación se reproduce en el ámbito laboral en las ocupaciones, en los sectores económicos y en el interior de las organizaciones (Anker 1997; Anker et al., 2003; Ibáñez, 2008; Cucco, 2013). De esta forma, al encontrarse el mercado de trabajo segregado en función del género, el sistema económico capitalista converge con el sistema patriarcal de género, que invisibiliza las labores de reproducción en el ámbito privado, generando situaciones de vulnerabilidad y exclusión (Benería, 2005, 2007; Ibáñez, 2008).

De hecho, en las profesiones consideradas como innatas a la condición femenina se valoran competencias y destrezas adquiridas en el proceso de socialización de género y, por lo tanto, no reducibles a un conocimiento transmisible a través de las instituciones académicas. Además, en ellas se ponen de manifiesto formas específicas de poder, la dominación patriarcal de género (Pinto, 1990; Maruani, 2002), que provoca una desvaloración de las cualidades o habilidades femeninas.

El fenómeno social de la segregación laboral según género se observa, con más o menos fuerza, a nivel mundial y sigue siendo importante en la actualidad. Hombres y mujeres no compiten en un mismo mercado de trabajo, sino que tienen condiciones laborales diferenciadas (Anker 1997; Anker et al., 2003; ILO 2012), en las que las mujeres ocupan puestos de menor cualificación y con menor reconocimiento pese a presentar niveles formativos y de cualificación similares e inclusive, en muchos casos, superiores (Molpeceres et al., 2013; Mariño y Rial, 2019).

Fruto de estas estructuras de dominación que generan mecanismos variados para producir la dominación de los varones sobre las mujeres (Anker 1997; Brunet y Alarcón, 2005), se mantienen desigualdades y discriminaciones tanto en la demanda como en la oferta (Ibáñez, 2008; Mariño y Rial, 2019). Los roles de género ligados a tal esquema de dominación inciden en la predisposición de las mujeres a inclinarse por formaciones típicamente femeninas y viceversa y hacen que las mujeres tiendan a agruparse en ciertas parcelas laborales y tener escasa presencia en otras como las tecnológicas (Fernández e Ibáñez, 2018) -segmentación horizontal- y en niveles de poder relativamente inferiores -segmentación vertical- debido a «barreras invisibles», que afectan, prioritariamente, al colectivo femenino para acceder a las mismas condiciones que los hombres (Carneiro, 2004). De estos obstáculos, a modo de ejemplo, los más conocidos son la «discriminación salarial» (Lago, 2002) y el denominado «techo de cristal» (Juesas, 2002). Esta situación, más allá de la discriminación producida, también genera rigideces en el mercado laboral e ineficiencia económica (Anker, 1997; Fernández e Ibáñez, 2018).

Más concretamente, en este sistema mujeres y hombres han sido descritos con unas características propias y diferenciadoras; así, las primeras han sido definidas a través de lo que Di Nicola (1991: 25), 
entre otras, han denunciado mediante lo que han denominado «la mística de la maternidad» ${ }^{6}$, donde se busca obtener un doble objetivo: primero, que asuman, de manera consentida, la sujeción de la mujer al hogar y, segundo, con la crianza, se convierten en salvaguarda y mantenedoras de las tradiciones.

Por el contrario, en los hombres se ha reafirmado la idea de que, al no tener la obligación de la crianza, ni cargas familiares, poseían una libertad que los convertía en «superior y poderoso»y, por eso, «ser trabajador y estar fuera de casa es un privilegio, el hombre no tiene carga. Por tanto, si es un privilegiado, no puede denunciar sus malestares, ya que de ese modo 'atentaría' contra sus privilegios» (Waisblat y Sáenz, 2011: 8). Se trata, por tanto, de un modelo de hombre manipulable y manipulado; construido y transmitido socialmente para lograr un fin «desde las lógicas de un ser omnipotente, activo, fuerte, y capacitado para enfrentarse con lo público de un modo privilegiado, con los códigos adecuados para el aprendizaje, competitivo, jerárquico y con lazos sociales precarios». (Waisblat y Sáenz, 2011: 9).

Sobre esta base, en líneas generales puede afirmarse que la incorporación de la mujer de manera masiva a la formación profesional y al mundo laboral no ha significado que se favorezca una ruptura de la concepción de los roles diferente de la existente ni el reparto equitativo en las tareas domésticas (Barberá, 2004). Por el contrario, como consecuencia del proceso de asalarización y mercantilización del empleo, las mujeres siguen realizando actividades que ya desempeñaban en la esfera doméstica en las organizaciones empresariales o públicas (Anker, 1997, Ibáñez, 2008). Sin olvidar los efectos de, la recesión «global» iniciada en 2008 y de las políticas de austeridad y de desreglamentación y flexibilización de los mercados de trabajo, como las reformas laborales de 2010 y 2012, que derivan en un empleo de peor calidad y mayores niveles de precariedad (temporalidad, estacionalidad, parcialidad) en el que se crea (Martín y Zarapuz, 2017; Aguado, 2019).

\section{Resultados: Formación Profesional y género}

Según Anker $(1997,1998)$ entre las teorías que explican la existencia y permanencia de la segregación ocupacional, pueden destacarse las siguientes: el nivel educativo, la experiencia de los trabajadores, las legislaciones vigentes, los prejuicios de los empleadores, el poder de las instituciones, la fuerte segmentación de ciertos mercados laborales y los estereotipos que la sociedad tiene contra determinados grupos (las mujeres). En los siguientes apartados, expondremos los resultados del análisis en relación con el primer caso, el nivel educativo de la FP.

\subsection{El crecimiento de la Formación Profesional}

Dados los menores niveles de participación en la FP en relación con los estudios universitarios (García y Lorente, 2015) y las diferencias con otros estudios secundarios postobligatorios ${ }^{7}$, con la promulgación de la LOGSE en 1990 y la legislación posterior ${ }^{8}$, se realiza una importante reestructuración en el sistema de enseñanza profesional, organizándola en ciclos formativos modulares, por áreas de conocimiento teórico-prácticas en relación con las diferentes familias profesionales para intentar modernizar su estructura, adaptarla a las nuevas necesidades sociales y productivas, homologables a la UE (García y Lorente, 2015).

$6 \quad$ Parodiando el texto de Friedan ([1963] 2016), La mistica de la feminidad.

7 En el seguimiento posterior de la investigación está previsto ahondar en la situación de la FP en relación con otros estudios y vías académicas de secundaria postobligatoria, como bachillerato. De este enfoque comparado pueden surgir interesantes aportaciones y una profundización de los resultados presentados, por ejemplo, respecto a las diferencias en la planificación de los estudios o entre los diversos perfiles del alumnado.

8 La normativa de referencia es la siguiente: Ley de Ordenación General del Sistema Educativo (LOGSE,1990), Ley Orgánica de las Cualificaciones y de la Formación Profesional (LOCFP, 2002), Ley Orgánica de Educación (LOE, 2006). Sobre esta normativa puede consultarse: García y Lorente (2015). 
El resultado ha sido que la evolución de la inscripción de alumnado en los CCFF en la última década, desde el inicio de la crisis, se ha incrementado de forma muy significativa?. Dicho aumento ha supuesto que casi se duplique la matrícula de la misma como puede observarse en el Gráfico 1 (Tabla I del anexo):

Gráfico 1. Evolución del alumnado matriculado en Foración Profesional

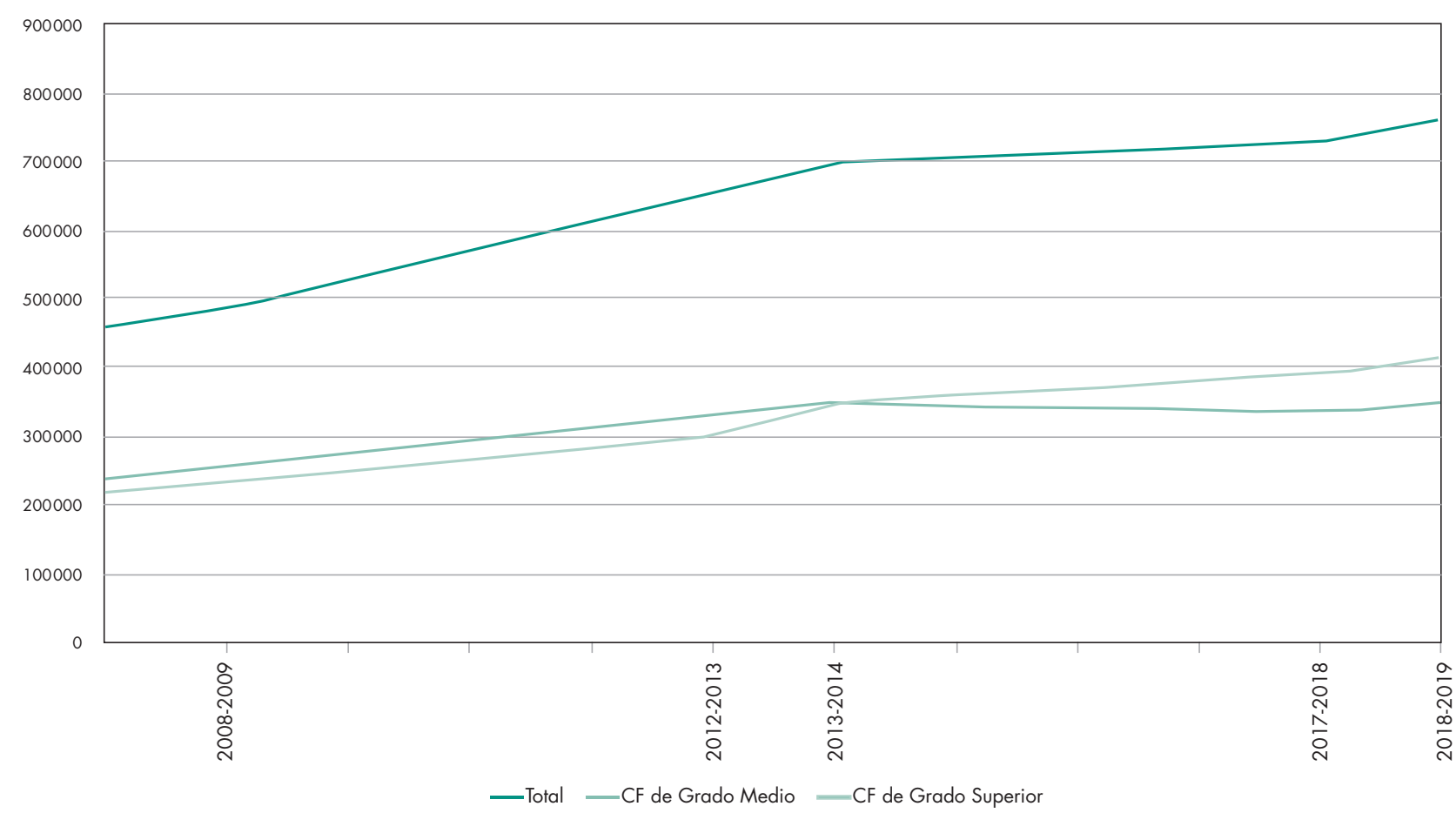

Fuente: Estadística de las Enseñanzas no universitarias. Subdirección General de Estadística y Estudios de MEyFP. Informes Datos y Cifras 2016-2017, 2018-2019 y 2019-2020 (MECD-MEyFP).

Elaboración propia.

En concreto, desde el curso 2007-2008 hasta el 2018-2019, la matriculación se incrementa en un $81,4 \%$ en total, un 46,4\% en los CCFF de Grado Medio (en adelante CFGM) y un 85,7\% en los CCFF de Grado Superior (en adelante CFGS). Debido a dicha evolución podría esperarse una posible distribución más equitativa del alumnado en cuestión de género o, cuanto menos, una posibilidad de romper con los estereotipos que provocan la segregación de género. Con otras palabras, la incorporación de las mujeres a la FP podría seguir una suerte de reversión del fenómeno de masculinización en esta formación y que acabe o reduzca la segregación de género del sistema educativo y, posteriormente, en el mercado laboral.

\subsection{Distribución por género de la FP}

La FP ha estado vinculada desde sus orígenes a los sectores industriales y tecnológicos, por lo que la presencia del alumnado femenino ha sido exigua durante décadas. Sin embargo, la representación de las mujeres y esta situación de invisibilidad ha mejorado notablemente, aunque, como veremos, no en todos los sectores (Rial et al., 2011; Mariño y Rial, 2019).

9 Sobre el crecimiento de la matriculación en FP puede consultarse: García y Lorente (2015) y Mariño y Rial (2019). 
Según el criterio de la Comisión Europea para calificar las profesiones mixtas, se consideran como tales «aquellas en las que la proporción tanto de hombres como de mujeres se sitúa entre el 40\% y el $60 \%$ (EC, 2014). De esta manera es posible hacer una extrapolación, aplicarla a los estudios formativos, y considerar que estos están más feminizados o masculinizados dependiendo de si existe una presencia femenina o masculina superior al $60 \%$.

Si seguimos este principio, en líneas generales no se puede afirmar que la FP sea una etapa educativa marcadamente masculinizada, en tanto que el rango entre el que oscila estaría dentro del 60-40 (Gráfico 2, Tabla II), puesto que la diferencia entre el alumnado masculino y el femenino tan solo asciende a 15,2 puntos porcentuales en el curso 2018-2019, un 57,6\% de hombres frente a un 42,4\% de mujeres. Por lo tanto, siguiendo la denominación aplicada por la Comisión Europea no se observaría un sesgo de género hacía un lado u otro y se podría considerar la FP, en general, como estudios mixtos, aunque existe una tendencia al descenso de las mujeres en los CFGM y un incremento en los CFGS).

Gráfico 2. Porcentaje de mujeres en los ciclos de FP

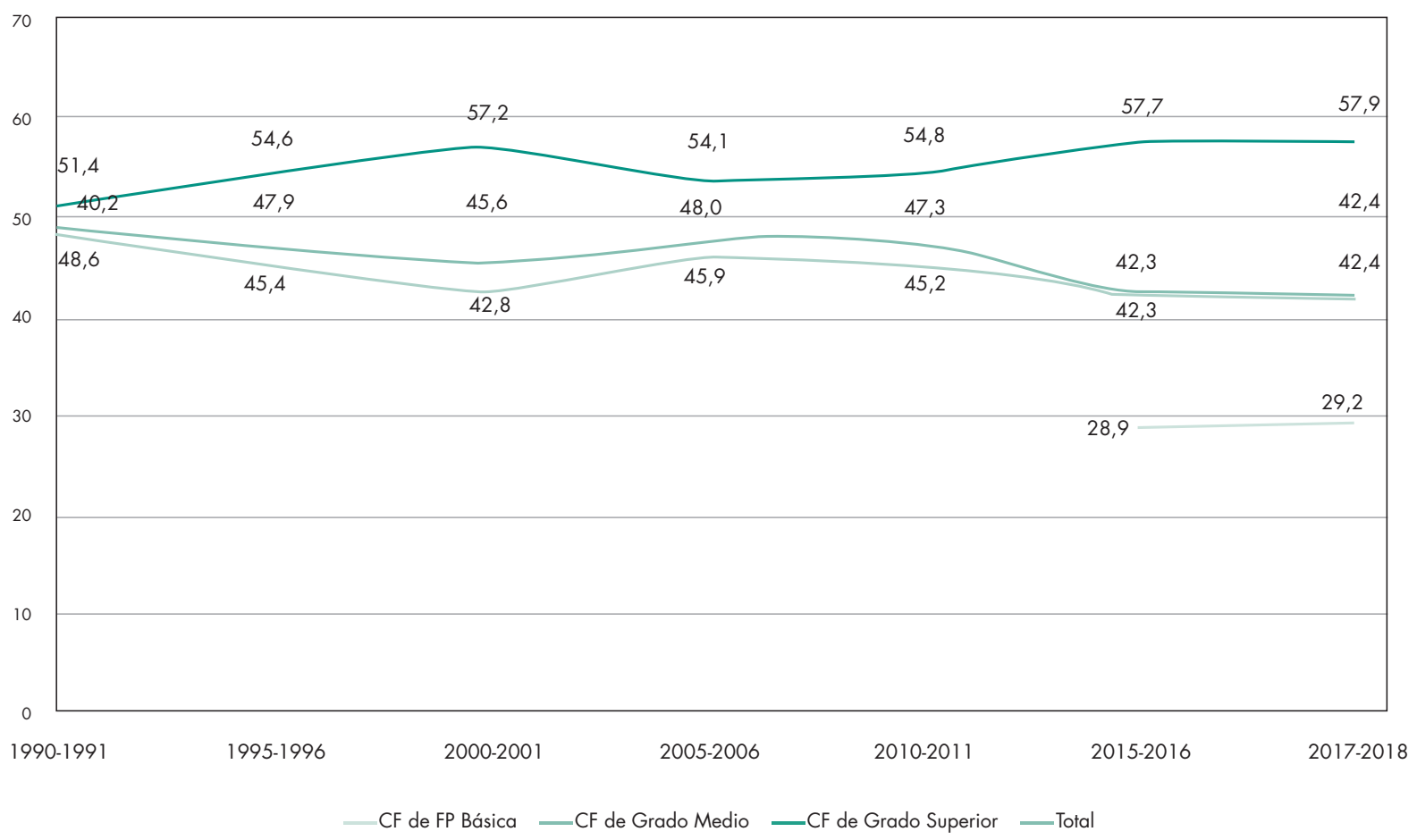

Fuente: Estadística de las Enseñanzas no universitarias. Subdirección General de Estadística y Estudios de MEyFP. Informes Datos y Cifras 2016-2017, 2018-2019 y 2019-2020 (MECD-MEyFP).

Elaboración propia. 
Sin embargo, cuando se profundiza más en el análisis, se aprecia una menor presencia masculina conforme se sube de nivel en este tipo de estudios. De esta manera, en la FP Básica el 70,8\% son hombres, mientras que en los CFGM se reducen al 57,9\% y en los CFGS siguen disminuyendo a un $54,6 \%$.

El alto índice de masculinización entre el alumnado de la FP Básica está en relación directa con el mayor abandono escolar temprano de los hombres ${ }^{10}$ y con el mayor rendimiento educativo de las jóvenes que se viene observando en las últimas décadas.

\subsection{Distribución por género según familias profesionales}

Para analizar profundizar en el análisis, de forma similar a la línea de investigación desarrollada por otros estudiosos de la materia (Rial et al., 2011; Urrea et al., 2018; Mariño y Rial, 2019), se ha acudido, asimismo, al estudio de la distribución por sexos de las diferencias vocacionales mediante la variable familia profesional ${ }^{11}$. De esta forma, como muestra la Tabla III, se observa una alarmante segregación horizontal de género en las mujeres en tanto que concentran su matrícula principalmente en cinco familias profesionales feminizadas: Administración y gestión, Imagen personal, Sanidad, Servicios socioculturales y a la comunidad y Textil, confección y piel. Dicha agrupación se traduce en que casi tres de cada cuatro mujeres se matriculan en los CCFF incluidos en las mismas.

Tabla III. Porcentaje de mujeres por familia profesional de FP

\begin{tabular}{|c|c|c|c|c|c|c|c|c|c|c|c|}
\hline \multirow[b]{2}{*}{$\%$ MUJERES } & \multicolumn{3}{|c|}{ CF FP BÁSICA } & \multicolumn{4}{|c|}{ CFGM } & \multicolumn{4}{|c|}{ CFGS } \\
\hline & $2015-2016$ & 2016-2017 & $2017-2018$ & 2011-2012 & $2015-2016$ & 2016-2017 & $2017-2018$ & $2011-2012$ & $2015-2016$ & 2016-2017 & $2017-2018$ \\
\hline Actividades Físicas y Deportivas & & & & 20,9 & 20,3 & 20,6 & 20,0 & 22,0 & 19,8 & 20,2 & 20,3 \\
\hline Administración y Gestión & 49,2 & 50,7 & 50,4 & 68,3 & 61,6 & 60,5 & 59,6 & 68,9 & 63,4 & 63,3 & 63,2 \\
\hline Agraria & 20,1 & 19,7 & 18,7 & 12,7 & 13,7 & 14,7 & 14,7 & 19,3 & 19,2 & 19,3 & 19,4 \\
\hline Artes Gráficas & 42,4 & 42,2 & 42,2 & 40,4 & 34,8 & 35,3 & 35,8 & 44,7 & 52,3 & 47,3 & 46,3 \\
\hline Artes y Artesanías & & & & & & & & & 43,3 & 43,5 & 45,5 \\
\hline Comercio y Marketing & 52,7 & 53,7 & 53,7 & 62,1 & 55,9 & 53,8 & 51,7 & 50,5 & 48,2 & 48,2 & 48,4 \\
\hline Edificación y Obra civil & 12,7 & 8,6 & 6,9 & 5,3 & 15,0 & 20,1 & 21,3 & 29,8 & 32,4 & 31,0 & 30,7 \\
\hline Electricidad y Electrónica & 3,9 & 3,4 & 3,5 & 2,4 & 2,8 & 8,6 & 3,0 & 4,9 & 5,0 & 4,9 & 4,8 \\
\hline Energía y Agua & & & & & & & 7,5 & 10,7 & 10,2 & 9,8 & 8,5 \\
\hline Fabricación Mecánica & 3,0 & 2,5 & 2,2 & 2,8 & 2,8 & 3,0 & 3,3 & 8,5 & 9,5 & 8,7 & 9,4 \\
\hline Hostelería y Turismo & 37,4 & 39,4 & 38,9 & 37,1 & 38,0 & 37,8 & 37,8 & 60,3 & 57,4 & 56,3 & 56,0 \\
\hline Imagen Personal & 86,3 & 84,7 & 83,1 & 94,5 & 92,3 & 91,2 & 89,7 & 96,2 & 94,9 & 94,3 & 94,1 \\
\hline Imagen y Sonido & & & & 49,2 & 33,8 & 23,8 & 22,6 & 37,2 & 33,8 & 32,7 & 32,3 \\
\hline Industrias Alimentarias & 39,6 & 42,5 & 39,7 & 44,0 & 54,3 & 54,5 & 54,2 & 46,5 & 47,9 & 46,9 & 46,1 \\
\hline Industrias Extractivas & & & & & 3,4 & 3,6 & 4,8 & & & & \\
\hline Informática y Comunicaciones & 18,3 & 17,8 & 17,7 & 11,4 & 8,9 & 8,0 & 7,8 & 14,2 & 12,9 & 11,7 & 11,4 \\
\hline Instalación y Mantenimiento & 1,5 & 3,4 & 5,4 & 1,8 & 2,1 & 2,1 & 2,1 & 18,5 & 3,2 & 14,4 & 13,2 \\
\hline Madera, Mueble y Corcho & 8,5 & 8,5 & 10,4 & 4,9 & 5,6 & 7,2 & 9,0 & 15,3 & 23,0 & 21,4 & 20,9 \\
\hline Marítimo-Pesquera & & 3,4 & & 7,7 & 6,4 & 6,9 & 6,6 & 9,1 & 10,7 & 9,9 & 9,4 \\
\hline Química & & & & 58,7 & 57,0 & 56,1 & 55,0 & 53,6 & 51,0 & 50,2 & 50,4 \\
\hline
\end{tabular}

10 Según el Informe Datos y Cifras en el curso escolar 2018-2019 del MEyFP, España se sitúa en el segundo país de la Unión Europea en Abandono Escolar y el Abandono Escolar Temprano supone un porcentaje del 18,3\% del alumnado (y de estos, un 21,8\% son hombres).

11 Si se ahonda más en el análisis desagregado por ciclos en lugar de las familias, podría observarse un sesgo de género diferente del de la familia a la que pertenecen. En la continuación de la investigación se considerará esta unidad de análisis básica que, por motivos de espacio, no se aborda en el presente trabajo. 


\begin{tabular}{|c|c|c|c|c|c|c|c|c|c|c|c|}
\hline \multirow[b]{2}{*}{ \% MUJERES } & \multicolumn{3}{|c|}{ CF FP BÁSICA } & \multicolumn{4}{|c|}{ CFGM } & \multicolumn{4}{|c|}{ CFGS } \\
\hline & 2015-2016 & 2016-2017 & 2017-2018 & $2011-2012$ & $2015-2016$ & 2016-2017 & 2017-2018 & 2011-2012 & 2015-2016 & 2016-2017 & 2017-2018 \\
\hline Sanidad & & & & 77,9 & 72,1 & 72,3 & 73,2 & 74,7 & 72,9 & 73,7 & 75,0 \\
\hline Seguridad y Medio Ambiente & & & & & 6,9 & 10,4 & 7,0 & & 41,2 & 34,7 & 34,4 \\
\hline $\begin{array}{l}\text { Servicios Socioculturales } \\
\text { y a la Comunidad }\end{array}$ & 80,6 & 78,3 & 72,7 & 87,2 & 85,6 & 85,9 & 85,8 & 90,5 & 87,7 & 87,3 & 87,1 \\
\hline Textil, Confección y Piel & 54,9 & 54,3 & 60,3 & 86,6 & 83,4 & 82,3 & 81,9 & 69,2 & 87,4 & 86,2 & 85,0 \\
\hline $\begin{array}{l}\text { Transporte y Mantenimiento } \\
\text { de Vehículos }\end{array}$ & 1,6 & 1,9 & 2,1 & 2,0 & 2,1 & 2,4 & 2,8 & 2,7 & 2,9 & 2,8 & 3,2 \\
\hline Vidrio y Cerámica & 29,0 & 31,9 & 39,7 & 8,3 & 12,7 & 18,1 & 79,2 & 27,6 & 25,0 & 46,7 & 30,4 \\
\hline Total general & 28,9 & 29,2 & 29,2 & 44,7 & 43,1 & 43,3 & 43,3 & 50,2 & 47,6 & 47,3 & 47,4 \\
\hline
\end{tabular}

Fuente: Estadística de las Enseñanzas no universitarias. Subdirección General de Estadística y Estudios de MEyFP. Informes Datos y Cifras 2016-2017, 2018-2019 y 2019-2020 (MECD-MEyFP).

Elaboración propia.

En el caso de los varones se observa que su presencia es mayoritaria en 15 familias, pero su dispersión por todas ellas tan solo asciende a matricular a dos de cada tres hombres, aunque monopolizan los sectores industriales y tecnológicos como: de Electricidad y Electrónica, Energía y Agua, Fabricación Mecánica, Informática y Comunicaciones, Instalación y Mantenimiento, Madera, Mueble y Corcho, Marítimo-Pesquera, Seguridad y medio ambiente y Transporte, Mantenimiento de Vehículos. Lo cual quiere decir que su presencia en la FP está mucho más diversificada.

Por otra parte, puede afirmarse que solo habría seis familias profesionales mixtas; es decir, donde la presencia de hombres y mujeres oscila entre el $60 \%$ y el $40 \%$.

Si consideramos los datos del mercado laboral existe cierto reflejo de dicha situación en tanto que en 2011 tan solo el 18\% de la población trabajadora estaba en ocupaciones en las que había paridad y la amplia mayoría de la fuerza de trabajo desarrollaba ocupaciones muy segregadas (Maira, 2017).

En general, el peso porcentual de las alumnas desciende en comparación con 2011 en todos los niveles de FP, aunque los últimos cursos su peso se mantiene prácticamente igual. No obstante, un análisis pormenorizado muestra algunos cambios en las tendencias existentes.

En el caso de las familias feminizadas se perciben evoluciones divergentes. En la de Imagen Personal se aprecia una aun exigua trayectoria descendente en el porcentaje de mujeres matriculadas -y por lo tanto en un mayor peso de los hombres- desde 2011 tanto en los CCFF de FP básica como en los CFGM y los CFGS. En la familia de Administración y gestión se observa una evolución similar desde 2011 en los CFGM al igual que en los CFGS, aunque en este último caso, los últimos tres cursos analizados el peso porcentual de las mujeres se mantiene prácticamente igual. En la de Sanidad, la tendencia es descendente en comparación con 2011 pero, tanto en los CFGM como en los CFGS, se aprecia un aumento del porcentaje de mujeres en los últimos cursos. En la de Servicios Socioculturales y a la Comunidad ocurre lo mismo, aunque en la FP básica aumenta el peso de los hombres. Por último, en Textil, Confección y Piel, el peso de las mujeres se incrementa en los CCFF de FP básica, mientras que desciende desde 2011en los CFGM y en los CFGS.

En las familias tradicionalmente masculinizadas también encontramos evoluciones diferenciadas. Por ejemplo, en la de Edificación y Obra Civil baja el peso de las mujeres en la FP básica, pero se 
incrementa en los CFGM y se mantiene prácticamente igual en los CFGS. En Hostelería y Turismo, la presencia femenina desciende en la FP superior y se mantienen más o menos igual en el resto de niveles. En las Industrias Alimentarias, desde 2011 aumenta el peso de las mujeres en los CFGM y en los CFGS asciende ligeramente en todo el período, pero decrece ligeramente los últimos años. En Informática y Comunicaciones, al igual que en Química, la matrícula de mujeres presenta una trayectoria descendente en todos los niveles. Instalación y Mantenimiento se incrementa en la FP básica. Por último, en Madera, Mueble y Corcho las mujeres amplían su presencia, aunque en los CFGS se observa un ligero decrecimiento los últimos cursos.

En definitiva, con todas las precauciones por la divergencia de las tendencias según las diferentes familias, se observa un aumento de la matriculación de hombres en los ciclos feminizados sin que se incremente en general el peso de las mujeres en los masculinizados.

\section{Discusión sobre la segregación de género en formación profesional}

Esta distribución segregada por género del alumnado de FP, como muchos otros fenómenos sociales, tiene tanto unas causas explicativas de tal situación como unas consecuencias que marcarán su posterior recorrido laboral y vital. A continuación, para contextualizar la investigación, se realizan aportaciones exploratorias al debate sobre algunas explicaciones de las razones que llevan a las y los jóvenes a optar por unas familias profesionales u otras y las posibles implicaciones que se derivan de dicha elección siguiendo los datos del análisis anterior, así como a diferentes estudios de la materia.

\subsection{Algunas explicaciones del fenómeno analizado}

En primer lugar, la segregación de género puede atribuye al proceso de socialización al que se ve sometida la juventud desde el mismo momento de su nacimiento. En dicho desarrollo, la familia y el sistema educativo son agentes de socialización determinantes en la orientación hacia los estudios y el empleo (Igbo et al., 2015; Maira, 2017; Fernández e Ibáñez, 2018). Dicha socialización de género es tan fuerte que lleva a que unas y otros interioricen gustos, preferencias y expectativas pareciendo dicha toma de decisiones una elección libre.

Otra de las explicaciones que describen dicho fenómeno responde a la identidad de género. Ésta es la primera identidad que se construye, prácticamente desde el nacimiento, por la cual los y las jóvenes asumen roles sociales, perciben que existen empleos y estudios con género, y en manifestación de dicha identidad toman decisiones según el rol que se espera de ellos y ellas (Fernández, 2016), sobre todo en su proceso vital de construcción como individuo -adolescencia-, más allá de las transformaciones pedagógicas y políticas que se han hecho y se hacen en el trabajo educativo por la igualdad (Amorós, 2000, 2005; Santos, 2006). También en la FP, donde el tratamiento transversal de la educación en valores tiene escasa presencia en el currículo, llegando incluso a intensificarse las estructuras de género del terreno profesional (Mosteiro y Porto, 2017; Urrea et al., 2018).

Ambas cuestiones son el origen de la aparición de estereotipos de género (Igbo et al., 2015; Mosteiro y Porto, 2017), que sostienen los roles de «hombre mantenedor-proveedon» y de «mujer cuidadora» (Urrea et al., 2018; Fernández e Ibáñez, 2018). De esta forma, se atribuyen competencias a mujeres con una clara tendencia a opciones laborales que prolongan las tareas domésticas y de cuidados -trato con las personas, perfeccionismo, afectividad, limpieza, etc.- (Maira, 2017). 
Asimismo, estos estereotipos inciden en que la tendencia formativa «natural» de los hombres es el ámbito científico-tecnológico y el de las mujeres el de las letras y las humanidades (Ibáñez, 2008; Rial et al., 2011; Fernández, 2016; Urrea et al., 2018; Mariño y Rial, 2019).

En este sentido, los roles y estereotipos de género están interrelacionados con la elección de las trayectorias formativas (Fernández e Ibáñez, 2018), reproduciendo la segregación sectorial-horizontal y laboral-vertical por familias profesionales según género (Mariño y Rial, 2019).

Un efecto derivado de las anteriores explicaciones, es la elección de estudios. Sobre todo, las jóvenes eligen en función de las mayores expectativas de integración laboral (Aguado, 2016; Mariño y Rial, 2019), son más pragmáticas a la hora de elegir (Cerdà et al., 201), cursando determinados estudios que les permitirán acceder al mercado laboral más fácilmente.

Pero, en dicha elección también son determinantes las sanciones familiares y sociales para los y las jóvenes que se separan del rol esperado o transgreden el orden social. Preceptos que son más severos en el caso de las jóvenes (Maira, 2017). Estudios como el de Mosteiro y Porto (2017), muestran como el alumnado masculino de FP ha interiorizado más que el alumnado femenino «ciertos estereotipos de género cuando aluden a la idoneidad de una actividad laboral u otra dependiendo del sexo y cuando se refieren a las competencias que es necesario dominar para realizar ciertas actividades asociadas a un tipo de trabajo determinado» (: 163). Por lo tanto, puede afirmarse que la elección de estudios, realmente se trata de una elección condicionada.

Asimismo, en la decisión de elegir la formación enfocada a oficios dominados por hombres por parte de las mujeres hay otros factores y barreras, tanto en el acceso como durante el aprendizaje, que se traducen en una brecha de género relacionada, entre otros, con la probabilidad de menores tasas de graduación (Berik et al., 2011), una desvinculación física y emocional durante la formación (Carcía et al., 2013) o el sufrir chanzas y ridiculización (Taylor et al., 2015). Como muestran Taylor et al., (2015), en la participación en estudios masculinizados influyen los programas de aprendizaje de secundaria y las influencias externas pero, sobre todo, el apoyo de las familias, especialmente, tener un padre trabajando en este oficio.

\subsection{Implicaciones del sesgo de género en las opciones formativas}

La elección de los estudios en las mujeres sigue estando condicionada, en parte, por los prejuicios sociales a que éstas desempeñen determinadas ocupaciones. Hemos visto como existe una concentración femenina en torno a cinco familias profesionales orientadas hacia los servicios, con un marcado sesgo de género, mientras que, asimismo, existe una atomización masculina por el resto de familias profesionales, aunque concentradas en la industria, la construcción y las nuevas tecnologías.

En este sentido, estamos de acuerdo con Ibáñez (2008: 109) en la afirmación de que las personas trabajadoras con FP «tienen menos probabilidades que el conjunto de la población de trabajar en ocupaciones no segregadas, y mayores de estar en ocupaciones masculinas o femeninas». Esta distinta exigencia de cualificaciones según se trate de trabajos feminizados y/o masculinizados anticipa una clara desventaja en términos de condiciones de trabajo (Fernández, 2016).

En este sentido, la concentración femenina en pocas familias profesionales supone menores opciones laborales y mayor precariedad laboral y vital, mientras que la dispersión masculina supone lo contrario: mayores opciones laborales y mejores condiciones laborales. 
En cuanto a las menores opciones laborales, responden a la concentración en cinco familias profesionales, lo cual reduce el abanico de opciones de empleo (en términos de oficios y profesiones) y refuerza la segregación de género. También a menor empleo en volumen (las profesiones técnicas y productivas son las que demandan mayor empleo).

Por su parte, la mayor precariedad laboral y vital viene dada por el hecho de que «la tecnología sea todavía un mundo de hombres» (Fernández e Ibáñez, 2018: 118) y la casi exclusiva presencia de la mujer en el sector servicios, marcado por peores condiciones de trabajo: mayor temporalidad, tiempo parcial, bajos salarios, etc., que, en particular, penaliza a las mujeres; tal y como puede observarse en la Encuesta Anual de Estructura Salarial (EAES) del INE, la brecha salarial en este sector es de aproximadamente un $25 \%$ en actividades sanitarias y servicios sociales o comercio ${ }^{12}$.

Además, en las profesiones STEM ${ }^{13}$, donde se produce una subrepresentación femenina, se da una situación de mayores salarios y mejores condiciones de trabajo en general. Así, según la EAES las mujeres, en la industria, cobran un 14\% más de lo que perciben en el sector servicios, generándose lo que podríamos denominar brecha salarial intragénero.

Como corolario, esa mayor precariedad se traduce en otra brecha: la brecha en las pensiones, generada por las menores cotizaciones producto de la situación de precariedad laboral y, también, por la maternidad -que provoca entradas y salidas del mercado laboral-, con consecuencias negativas directas sobre el importe de las pensiones futuras. De hecho, se estima que la brecha de género en las pensiones asciende a unos $6000 €$ anuales (CCOO, 2018).

\section{Conclusiones y propuestas}

Como se observa en el análisis expuesto, como consecuencia del crecimiento que ha sufrido el sector servicios, se han experimentado cambios en la composición de la FP y en relación al alumnado que se matricula en los distintos CCFF. Pese a ello, en general, el peso porcentual de las alumnas desciende en comparación con 2011 en todos los niveles de FP, aunque en los últimos cursos su peso se mantiene prácticamente igual.

Puede afirmarse que la FP es una etapa educativa con una clara segregación horizontal de género que se manifiesta en una concentración femenina en torno a cinco familias profesionales con un marcado sesgo de género, vinculadas estrechamente a las tareas de cuidados (sanidad, servicios sociales, educación) y a los estereotipos de género (imagen personal, textil, etc.), junto a una dispersión masculina por el resto de familias profesionales, aunque concentradas en la industria, la construcción y las nuevas tecnologías.

Desde un análisis pormenorizado, con todas las precauciones que hay que tener por la divergencia de las trayectorias según las diferentes familias profesionales, en los últimos años se observa un aumento de la matriculación de hombres en los ciclos feminizados sin que se incremente en general el peso de las mujeres en los masculinizados, lo que no permita hablar de una tendencia a la reducción del sesgo de género en este ámbito.

Las causas de dicha segregación de género pueden encontrarse en el proceso de socialización que experimentan los y las jóvenes, en la construcción de la identidad de género que les lleva a percibir que los

12 https://www.ine.es/ss/Satellite?L=es_ES\&c=INESeccion_C\&cid=1259925408327\&p=1254735110672\&pagename=ProductosYServicios\%2FPYSLayout

13 Science, Technologies, Engineering and Mathematics. 
estudios y oficios tienen género y, derivado de todo ello, en la elección de estudios con una clara influencia de la familia y el sistema educativo, pero, también, para el caso de las jóvenes por las mayores expectativas laborales.

Dicha situación genera, a posteriori, unas consecuencias negativas para las jóvenes que desemboca en una situación de desigualdad en tanto que les reduce el abanico de opciones laborales y les obliga a insertarse laboralmente en sectores y profesiones que padecen mayor precariedad laboral y vital, lo que, a su vez, se traduce en un incremento de la brecha salarial y de las pensiones.

Retomando la idea «campos» de Bourdieu ([1979] 1988, [1980] 1991), indicada al inicio del texto, ante esta situación de desigualdad hacia nuestras jóvenes en el espacio estructurado de relaciones objetivas de acción e influencia en la esfera de la formación y entre ésta y el ámbito laboral, planteamos algunas actuaciones que muestran el potencial de las instituciones educativas, por su situación en la estructura de la distribución de las diferentes formas de poder, para revertir dicha situación o, cuanto menos, minimizarla.

Siempre teniendo en cuenta el concepto de «habitus vocacional» que pone de manifiesto que el estudiantado se orienta a un conjunto particular de predisposiciones, tanto idealizadas como realizadas. Este «habitus» puede reproducir las desigualdades sociales y de género. Sin embargo, las culturas vocacionales en las que están inmersas las estudiantes también les transforman reforzando o desarrollando estos factores según las demandas del puesto de trabajo, por lo que implica desarrollar un «sentido» de cómo ser de forma apropiada para cada persona y, también, la «sensibilidad», los sentimientos y la ética necesarios para ello (Colley et al., 2003).

En primer lugar, es urgente un diagnóstico serio sobre la cultura de género del alumnado de FP para incrementar las oportunidades profesionales de todos, hombres y mujeres, junto con el compromiso de todas las entidades encargadas de la formación (Urrea et al., 2018), administraciones gubernamentales y centros educativos.

En segundo lugar, hay que romper con los estereotipos de género. El profesorado tiene capacidad para influir en el alumnado y potenciar la igualdad de género, con lo cual sería deseable su implicación para que orientaran su quehacer docente hacia la igualdad.

Asimismo, se deben mostrar las aportaciones de las mujeres al progreso y al desarrollo económico y social. Pero también visibilizar a aquellas personas que han roto con la norma social esperada de ellas según su condición de género.

Para ello, es positivo realizar campañas publicitarias contra los roles de género. Ya ha habido algunas como, por ejemplo, las realizadas por el Ayuntamiento de València contra los juegos y juguetes sexistas ${ }^{14} \mathrm{O}$ por la Viceconselleria d'Igualtat de la Generalitat Valenciana contra los estereotipos LGTBI ${ }^{15}$.

Por otro lado, es preciso fomentar la capacitación y sensibilización del profesorado en temas de igualdad y equidad entre los géneros, para que puedan adoptar una toma de conciencia respecto a la importancia de la igualdad de género y, también, de no reproducir los estereotipos de género en la práctica y el proceso pedagógico.

14 http://www.valencia.es/valencia/noticias/NOTICIA_057281?lang=1\&seccion=5\&temId=17\&nivel=5_2_17

15 http://www.inclusio.gva.es/es/trenca-l-estereotip 
Se precisa una Orientación Educativa para la Igualdad en todas las etapas educativas, con especial énfasis en las primeras (primaria y secundaria), donde por edad, precisan de información concreta sobre temas de sexualidad y relación con el otro sexo y su correlación con los estereotipos de género en los empleos futuros.

En la FP, sería interesante prestar atención a los materiales didácticos y promover entre el alumnado femenino los CCFF que tienen mayor presencia masculina y con una mejor inserción laboral. De esta manera, se romperían los estereotipos laborales de género que en la actualidad perduran y podría conseguirse que las jóvenes accedieran a profesiones con mejores condiciones de trabajo. En este sentido, en la Comunitat Valenciana, desde hace varios cursos, la Conselleria d'Educació está intentando motivar el acceso de las mujeres a una serie de CCFF masculinizados (Electricidad y electrónica, Energía y agua, Fabricación mecánica, etc.) a través de incentivos para alumnas que se matriculen en ellos ${ }^{16}$.

Por último, se precisa seguir promoviendo la igualdad de género dentro de los CCFF extendiéndolo a todo el Estado español. Existen algunas experiencias al respecto como, por ejemplo, en el IV Plan Vasco de FP o la Guía para la incorporación de la perspectiva de género en el currículum del Departamento de Educación del Gobierno Vasco (Arraiz et al., 2015) ${ }^{17}$; el Proyecto SKOLAE de coeducación para la igualdad en el aula el Gobierno de Navarra, premiado por la UNESCO ${ }^{18}$; el Proyecto Orienta que busca abordar la brecha de género en FP de la Federación de Enseñanza de CCOO (Arroyo, 2017) ${ }^{19}$; o el proyecto \#Pioneras FP de HETEL Euskadi, que tiene como objetivo animar a las mujeres a romper moldes y realizar estudios de FP industrial ${ }^{20}$.

En cualquier caso, todas estas propuestas, están en relación con los resultados obtenidos en estudios de similares características (Urrea et al., 2018: 29) donde, en definitiva, se considera necesaria:

La puesta en marcha de actuaciones que permitan el reconocimiento y transformación de este tipo de creencias entre el alumnado de Formación Profesional. Los centros educativos no pueden permanecer ajenos a los desafíos que plantea la sociedad actual y, especialmente, a los obstáculos y barreras que la mujer ha de superar en el contexto laboral.

\section{Referencias bibliográficas}

Aguado, Empar (2016): "Mujeres en la estiba. El caso de las estibadoras portuarias en el puerto de Valencia”. Arxius de Ciències Socials, 35, 123-138.

Aguado, Juli Antoni (2019): "La relación entre el mercado de trabajo y las políticas activas de empleo y desarrollo local en el País Valenciano (1997-2017): un estudio en curso". Lan Harremanak. Revista de Relaciones Laborales, 42, 1-25. DOI: https://doi.org/10.1387/lan-harremanak.21137.

Amorós, Cela (2000). Feminismo y filosofía. Madrid: Síntesis.

\footnotetext{
$16 \mathrm{http} / /$ www.ceice.gva.es/en/web/formacion-profesional/ayudas-alumnas-de-determinados-ciclos-formativos

17 https://www.euskadi.eus/contenidos/enlace/fp/es_evalua/adjuntos/guia.pdf

18 https://www.educacion.navarra.es/documents/27590/1325202/Libro+SKOLAE+CAST+web.pdf/1ba58d8b-d13b-4f2d-0fc1-9c5e99d5946e; https://www.educacion.navarra.es/web/dpto/skolae-profesorado

19 https://fe.ccoo.es/eab191334cf62c350f316caa41b422bf000060.pdf

20 https://hetel.eus/index.php/es/noticias/650-en-marcha-la-campana-pionerasfp-abantean; https://hetel.eus/index.php/es/aitzindariakfp
} 
Amorós, Celia (2005). La gran diferencia y sus pequeñas consecuencias... para la lucha de las mujeres. Valencia: Cátedra.

Anker, Richard (1997): "La segregación ocupacional entre hombres y mujeres. Repaso de las teorías". Revista Internacional del Trabajo, 116 (3), 343-370.

Anker, Richard (1998). Gender and Jobs: Sex Segregation of Occupations in the World. Geneva: International Labour Office.

Anker, Richard; Melkas, Helinä y Korten, Ailsa (2003): "Gender Based Occupational Segregation in the 1990's". Working Paper, 16. In Focus Programme on Promoting the Declaration on Fundamental Principles and Rights at Work. International Labour Office.

Arroyo, Lidia; Gil, Miguel Ángel; Heredero, Carmen; Maira, Mar; Otaegui, Amaia; Plaza, Pedro; Ponce, Yolanda y Rodero, Isabel (2017). Proyecto Orienta: Segregación de género en el sector industrial: Estrategias educativas y laborales. Madrid: CCOO de Industria y Federación de Enseñanza de CCOO.

Arraiz, Begoña; Careaga, Haizea; Carramiñana, Susana y Oskoz, José Miguel (2015). Guía para la incorporación de la perspectiva de género en el curriculum y en la actividad docente de las enseñanzas de régimen especial y de formación profesional: propuestas concretas para Formación y Orientación Laboral (FOL) y Empresa e Iniciativa Emprendedora (EIE). Vitoria-Gasteiz: Servicio Central de Publicaciones del Gobierno Vasco.

Barberá, Ester (2004): "Diversidad de género, igualdad de oportunidades y entornos laborales". Revista de Economía Pública, Socialy Cooperativa, 50, 37-53.

Berik, Günseli; Bilginsoy, Cihan y Williams, Larry S. (2011). "Gender and Racial Training Gaps in Oregon Apprenticeship Programs.” Labor Studies Journal, 36(2), 221-244. DOI: https://doi.org/10.1177/0160449X10396377.

Benería, Lourdes (2005). Género, desarrollo y globalización. Por una ciencia económica para todas las personas. Barcelona: Hacer.

Benería, Lourdes (2007): “Trabajo productivo/reproductivo, pobreza, y políticas de conciliación en América Latina: consideraciones teóricas y prácticas" en Judith Astelarra (coord.): Género y cohesión social. Madrid: Fundación Carolina.

Bourdieu, Pierre ([1979] 1988). La distinción. Criterio y bases sociales del gusto. Madrid: Taurus.

Bourdieu, Pierre ([1980] 1991). El sentido práctico. Madrid: Taurus.

Bourdieu, Pierre y Wacquant, Loïc (2005). Una invitación a la sociología reflexiva. Buenos Aires: Siglo XXI.

Brunet, Ignasi y Alarcón, Amado (2005): "Mercado de Trabajo y Familia". Revista de Investigaciones Políticas y Sociológicas (RIPS), 4 (2), 115-129.

Carneiro, Manuel (2004). La Responsabilidad Social Corporativa interna: la "nueva frontera" de los Recursos Humanos. Madrid: ESIC.

CCOO (2018). La brecha de género en el sistema de protección social (desempleo y pensiones). Madrid: Secretaría de Políticas Públicas y Protección Social CS de CCOO. 
Cebrián, Inmaculada y Moreno, Gloria (2008): “La situación de las mujeres en el mercado de trabajo español: desajustes y retos”. Economía industrial, 367, 121-137.

Cerdà, Antoni; Salvà, Francesca y Calvo, Aina (2016). "Género y elección profesional en los ciclos formativos de grado medio”. XII Congreso Español de Sociologia. Gijón, julio.

Cobo, Rosa (2011). Hacia una nueva politica sexual: las mujeres ante la reacción patriarcal. Madrid: Los Libros de la Catarata.

Colley, Helen; James, David; Diment, Kim y Tedder, Michael (2003): "Learning as Becoming in Vocational Education and Training: Class, Gender and the Role of Vocational Habitus". Journal of Vocational Education and Training, 55 (4), 471-498. DOI: https://doi.org/10.1080/13636820300200240.

Cucco, Mirtha (2013): “¿Engranajes que se desplazan, espacios que se abren? Superando el rol de proveedor o nuevas versiones renovadas". Jornadas sobre Cuestiones de género: Los aportes ProCC. De la masculinidad begemónica a las masculinidades. La Habana, julio.

Di Nicola, Giulia Paola (1991). Reciprocidad hombre/mujer. Igualdad y Diferencia. Madrid: Narcea.

EC (2014). Report on Progress on Equality Between Women and Men in 2013. Brussels: European Commission, 14.4.2014. SWD (2014) 142 final.

Fernández, Ana Belén (2016): “Coeducación y profesiones masculinizadas. El papel del sistema educativo como agente de socialización”. XII Congreso Español de Sociologia. Gijón, julio.

Fernández, Ana Belén e Ibáñez, Marta (2018): “Más mujeres en los estudios de Informática: una propuesta desde el departamento de formación y orientación laboral”. Revista de Sociología de la Educación (RASE), 1 (1), 116-134. DOI: http://dx.doi.org/10.7203/RASE.11.1.10624.

Friedan, Betty ([1963] 2016). La mistica de la feminidad. Valencia: Cátedra.

García, Maribel; Casal, Joaquim; Merino, Rafael y Sánchez, Albert (2013). "Itinerarios de abandono escolar y transiciones tras la Educación Secundaria Obligatoria”. Revista de educación, 361, 65-94. DOI: https://doi.org/10.4438/1988-592X-RE-2011-361-135.

García, Enrique y Lorente, Rocío (2015): "Recorrido por la imagen social de la Formación Profesional: un camino hacia su revalorización". Revista Española de Educación Comparada, 26, 119-134. DOI: https://doi.org/10.5944/ reec.26.2015.14270

Ibáñez, Marta (2008): “La segregación ocupacional por sexo a examen: características personales, de los puestos y de las empresas asociadas a las ocupaciones masculinas y femeninas". Revista Española de Investigaciones Sociológicas (REIS), 123, 87-122.

Igbo, J. N., Onu, V. C., \& Obiyo, N. O. (2015): "Impact of Gender Stereotype on Secondary School Students' self-concept and academic achievement”. Sage Open, 5(1), 1-10. DOI: https://doi. $\operatorname{org} / 10.1177 / 2158244015573934$.

ILO (2012). Global Employment Trends for Women 2012. Geneva: International Labour Organization. 
Juesas, Martha (2002). Corbatas y tacones: cómo se relacionan hombres y mujeres en el trabajo. Madrid: Temas de Hoy.

Lago, Ignacio (2002): "La discriminación salarial por razones de género: un análisis empírico del sector privado en España”. Revista Española de Investigaciones Sociológicas (REIS), 98, 171-196.

Mariño, Raquel y Rial, Antonio (2019): "Balance de las necesidades formativas demandadas por mujeres que cursan o que han cursado ciclos de FP en familias profesionales masculinizadas: el caso de Galicia". Educar, 55 (1), 251-272.

Maira, María del Mar (2017): “La segregación horizontal por género y sus consecuencias en la ocupación masculinizada de mecánico/a en el subsector de reparación de vehículos en España”. Laboreal, 13 (1), 9-23. DOI: https://doi.org/10.4000/laboreal.1796.

Martín, Aurelia (2006). Antropología del género. Madrid: Cátedra.

Martín, Carlos y Zarapuz, Luis (2017). El crecimiento se basa en la desigualdad. Situación económica de España y PGE-2017. En clave de Economía. Madrid: Gabinete económico de CCOO, junio.

Maruani, Margaret (2002). Trabajo y el empleo de las mujeres. Madrid: Fundamentos.

Molpeceres, Laura; Ongil, Marta; Henar, Leticia y Rodríguez, Maite (2013). Formación profesional de las mujeres y nuevos yacimientos de empleo. Madrid: Enred - Instituto de la Mujer, Ministerio de Sanidad, Servicios Sociales e Igualdad.

Mosteiro, Ma Josefa y Porto, Ana María (2017): “Análisis de los estereotipos de género en alumnado de Formación Profesional: diferencias según sexo, edad y grado”. Revista de Investigación Educativa, 35 (1), 151-165. DOI: https://doi.org/10.6018/rie.35.1.257191.

Pinto, Josiane (1990): "Une relation enchanteé, la secrètaire et son patron”. Actes de la recherche en sciences sociales, $84,32-48$.

Rial, Antonio F.; Mariño, Raquel y Rego, Laura (2011): "La situación de la mujer como alumna de formación profesional inicial y como profesional en el mercado laboral”. Revista Iberoamericana de Educación, $54(6), 1-13$.

Santos, Ma Ángeles (Coord.) (2006). El harén pedagógico. Perspectiva de género en la organización escolar. Barcelona: Graó

Taylor, Alison; Hamm, Zane y Raykov, Milosh (2015). "The experiences of female youth apprentices in Canada: just passing through?”. Journal of Vocational Education and Training, 67 (1), 93-108. DOI: https://doi.org/10.1080/13636820.2014.896404

Tubert, S. (Ed.). (2003). Del sexo al género. Los equívocos de un concepto. Valencia: Cátedra.

Urrea, María Encarnación; Fernández, Aitana; Aparicio, María del Pilar y Hernández-Amorós, María J. (2018): “La cultura de género del alumnado de Formación Profesional en el ámbito laboral”. International Journal of Developmental and Educational Psychology - INFAD Revista de Psicología, 2(1), 23-32.

Waisblat, Alfred y Sáenz, Ana (2011): "La construcción socio-histórica de la existencia. Patriarcado, capitalismo y desigualdades instaladas". Jornadas sobre "Roles masculino y femenino a debate”. Bilbao, enero. 


\section{Anexo}

\section{Tabla I. Evolución del alumnado matriculado en Formación Profesional}

\begin{tabular}{lcccccc} 
& $2007-2008$ & $2008-2009$ & $2012-2013$ & $2013-2014$ & $2017-2018$ & $2018-2019$ \\
CF de FP Básica & & & & 72186 & 74009 \\
\hline CF de Grado Medio & 239559 & 253516 & 332495 & 350250 & 338112 & 350820 \\
\hline CF de Grado Superior & 222933 & 233377 & 303063 & 348444 & 393531 & 413935 \\
\hline Total & 462492 & 486983 & 661047 & 698694 & 804829 & 838764 \\
\hline
\end{tabular}

Fuente: Estadística de las Enseñanzas no universitarias. Subdirección General de Estadística y Estudios de MEyFP. Informes Datos y Cifras 2016-2017, 2018-2019 y 2019-2020 (MECD-MEyFP).

Elaboración propia.

Tabla II. Porcentaje de alumnado de FP por género

\begin{tabular}{|c|c|c|c|c|c|c|c|}
\hline CF de FP Básica & $1990-1991$ & $1995-1996$ & $2000-2001$ & $2005-2006$ & 2010-2011 & 2015-2016 & 2017-2018 \\
\hline Hombres & & & & & & 43993 & 51078 \\
\hline Mujeres & & & & & & 17916 & 21102 \\
\hline Total & & & & & & 61909 & 72180 \\
\hline$\%$ & $1990-1991$ & $1995-1996$ & $2000-2001$ & $2005-2006$ & 2010-2011 & $2015-2016$ & 2017-2018 \\
\hline Hombres & & & & & & 71,1 & 70,8 \\
\hline Mujeres & & & & & & 28,9 & 29,2 \\
\hline CF de Grado Medio & $1990-1991$ & $1995-1996$ & $2000-2001$ & $2005-2006$ & $2010-2011$ & $2015-2016$ & 2017-2018 \\
\hline Hombres & 1467 & 16096 & 109483 & 124487 & 158733 & 188772 & 184942 \\
\hline Mujeres & 1389 & 13361 & 81973 & 105687 & 130835 & 138362 & 134327 \\
\hline Total & 2856 & 29457 & 191456 & 230174 & 289568 & 327134 & 319269 \\
\hline$\%$ & $1990-1991$ & $1995-1996$ & $2000-2001$ & 2005-2006 & $2010-2011$ & 2015-2016 & 2017-2018 \\
\hline Hombres & 51,4 & 54,6 & 57,2 & 54,1 & 54,8 & 57,7 & 57,9 \\
\hline Mujeres & 48,6 & 45,4 & 42,8 & 45,9 & 45,2 & 42,3 & 42,1 \\
\hline CF de Grado Superior & $1990-1991$ & $1995-1996$ & $2000-2001$ & 2005-2006 & $2010-2011$ & 2015-2016 & 2017-2018 \\
\hline Hombres & 2284 & 16053 & 95363 & 108249 & 133944 & 170566 & 190383 \\
\hline Mujeres & 2256 & 16232 & 89688 & 109006 & 132068 & 144041 & 158332 \\
\hline Total & 4540 & 32285 & 185051 & 217255 & 266012 & 314607 & 348715 \\
\hline$\%$ & $1990-1991$ & $1995-1996$ & $2000-2001$ & $2005-2006$ & $2010-2011$ & $2015-2016$ & 2017-2018 \\
\hline Hombres & 50,3 & 49,7 & 51,5 & 49,8 & 50,4 & 54,2 & 54,6 \\
\hline Mujeres & 49,7 & 50,3 & 48,5 & 50,2 & 49,6 & 45,8 & 45,4 \\
\hline Total FP & 1990-1991 & $1995-1996$ & $2000-2001$ & $2005-2006$ & $2010-2011$ & 2015-2016 & 2017-2018 \\
\hline Hombres & 3751 & 32149 & 204846 & 232736 & 292677 & 403331 & 426403 \\
\hline Mujeres & 3645 & 29593 & 171661 & 214693 & 262903 & 300319 & 313761 \\
\hline Total & 7396 & 61742 & 376507 & 447429 & 555580 & 703650 & 740164 \\
\hline$\%$ & $1990-1991$ & $1995-1996$ & $2000-2001$ & 2005-2006 & $2010-2011$ & 2015-2016 & 2017-2018 \\
\hline Hombres & 50,7 & 52,1 & 54,4 & 52,0 & 52,7 & 57,3 & 57,6 \\
\hline Mujeres & 49,3 & 47,9 & 45,6 & 48,0 & 47,3 & 42,7 & 42,4 \\
\hline
\end{tabular}

Fuente: Estadística de las Enseñanzas no universitarias. Subdirección General de Estadística y Estudios de MEyFP. Informes Datos y Cifras 2016-2017, 2018-2019 y 2019-2020 (MECD-MEyFP).

Elaboración propia. 


\section{Notas biográficas}

Juli Antoni Aguado Hernández es Doctor en Sociología por la Universitat de València y Licenciado en Ciencias Políticas y Sociología por la Universidad Nacional de Educación a Distancia. También ha realizado posgrados sobre Desarrollo Local. Profesor asociado en el Departament de Sociologia i Antropologia Social de la Universitat de València y Agente de Desarrollo Local en la administración local. Sus principales líneas de investigación versan sobre: Movimientos sociales y desobediencia civil, Participación ciudadana y Planificación estratégica y sobre Desarrollo local en relación con Técnicas de investigación social, Responsabilidad Social Corporativa y Políticas de Empleo.

Francisco Javier Cano Montero es Licenciado en Ciencias del Trabajo por la Universitat de València y Diploma de Estudios Avanzados en Ciencias del Trabajo por la Universitat de València. Profesor asociado del Departament de Sociologia i Antropologia Social de la Universitat de València y profesor de secundaria -especialidad Formación y Orientación Laboral- en la Conselleria d'Educació de la Generalitat Valenciana. Las líneas de investigación realizadas se han centrado en torno a la inserción profesional de los egresados en el Grado de RRHH y RRLL, así como al análisis de los estereotipos y las desigualdades de género que existen en la FP. Su actividad docente universitaria se ha centrado en los últimos años en la sociología de la educación y del trabajo.

María José Sánchez Pérez es Doctora en Ciencias de la Educación por la Universitat de València, Licenciada en Pedagogía por la Universitat de València y Licenciada en Psicopedagogía por la Universidad Nacional de Educación a Distancia. Profesora asociada del Departament de Mètodes d’Investigació i Diagnòstic Educatiu de la Universitat de València y profesora de secundaria -especialidad Orientación Educativa- en la Conselleria d'Educació de la Generalitat Valenciana. Las líneas de investigación desarrolladas se centran en competencias profesionales que desarrollan los orientadores educativos, orientación profesional y atención inclusiva. 